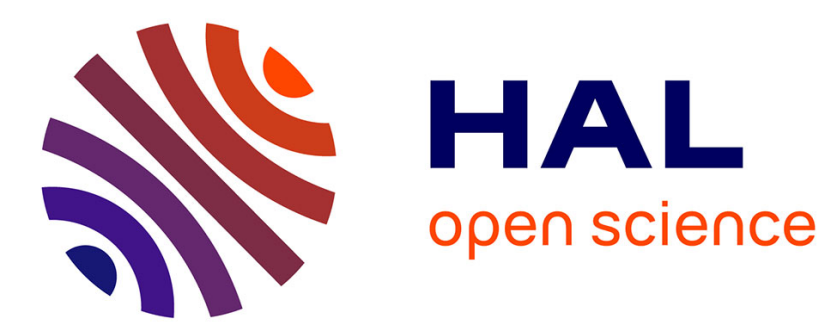

\title{
Species segregation in one-dimensional granular-system simulations
}

Filippo Pantellini, Simone Landi

\section{To cite this version:}

Filippo Pantellini, Simone Landi. Species segregation in one-dimensional granular-system simulations. European Physical Journal E: Soft matter and biological physics, 2008, 25 (2), pp.201-212. 10.1140/epje/i2007-10281-5 . hal-03090598

\section{HAL Id: hal-03090598 https://hal.science/hal-03090598}

Submitted on 29 Dec 2020

HAL is a multi-disciplinary open access archive for the deposit and dissemination of scientific research documents, whether they are published or not. The documents may come from teaching and research institutions in France or abroad, or from public or private research centers.
L'archive ouverte pluridisciplinaire HAL, est destinée au dépôt et à la diffusion de documents scientifiques de niveau recherche, publiés ou non, émanant des établissements d'enseignement et de recherche français ou étrangers, des laboratoires publics ou privés. 


\title{
Species segregation in one-dimensional granular system simulations
}

\author{
Filippo Pantellini ${ }^{1}$ and Simone Landi ${ }^{2}$ \\ 1 LESIA, Observatoire de Paris, CNRS, UPMC, Université Paris Diderot, 5 Place Jules Janssen, 92195 Meudon, France \\ 2 Dipartimento di Astronomia e Scienza dello Spazio, Largo Enrico Fermi 2, 50125 Firenze, Italy
}

Received 9 july 2007 and Received in finale form 16 November 2007

\begin{abstract}
We present one dimensional molecular dynamics simulations of a two species, initially uniform, freely evolving granular system. Colliding particles swap their relative position with a $50 \%$ probability allowing for the initial spatial ordering of the particles to evolve in time and frictional forces to operate. Unlike one dimensional systems of identical particles, two species one dimensional systems of quasi-elastic particles are ergodic and the particles' velocity distributions tend to evolve towards Maxwell-Boltzmann distributions. Under such conditions, standard fluid equations with merely an additional sink term in the energy equation, reflecting the non elasticity of the interparticle collisions, provide an excellent means to investigate the system's evolution. According to the predictions of fluid theory we find that the clustering instability is dominated by a non propagating mode at a wavelength of the order $10 \pi L / N \varepsilon$, where $N$ is the total number of particles, $L$ the spatial extent of the system and $\varepsilon$ the inelasticity coefficient. The typical fluid velocities at the time of inelastic collapse are seen to be supersonic, unless $N \varepsilon \lesssim 10 \pi$. Species segregation, driven by the frictional force occurs as a result of the strong temperature gradients within clusters which pushes the light particles towards the clusters' edges and the heavy particles towards the center. Segregation within clusters is complete at the time of inelastic collapse.
\end{abstract}

PACS. $45.70 . \mathrm{Mg}$ Granular flow: mixing, segregation and stratification - 45.50.Tn Collisions - 02.70.Ns Molecular dynamics and particle methods

\section{Introduction}

Granular materials are ubiquitous in the macroscopic world [1]. The consequence of the inelasticity of the collisions between grains is that the standard fluid equations used to describe a gas of atoms or molecules need to be modified. Even for the simplest systems, the mathematical expressions for the transport coefficients turn out to be much more involved than the expressions for a system of elastic particles. In addition, granular materials are often polydisperse. For example sand grains are generally characterized by a broad range of particle sizes and shapes. Thus, theoretical models and numerical simulations based on granular systems of identical particles may be too limited to describe a real system, even on a purely qualitative level. On the other hand, systems of identical particles have the non negligible advantage of being mathematically simple while retaining many important features of real dissipative systems.

One of the most spectacular consequences of the inelastic nature of collisions in a granular system is the so called inelastic collapse. In the absence of energy injection statistical fluctuations in an initially uniform system can lead some regions to cool faster than others producing linearly growing density fluctuations which can be described in the frame of continuum model theories $[2,3,4]$. In the non linear regime the high density regions collapse producing clumps of particles where the collision frequency grows to infinity $[5,6]$. The instability is a long wavelength instability which is hardly avoidable in freely evolving systems provided the number of particles in the system exceeds some minimum value which very much depends on the system's characteristics $[6,3]$

It is often acceptable to describe granular systems by means of fluid equations derived from the inelastic Boltzmann equation using the Chapman-Enskog procedure [7, 8, 9]. Hydrodynamic models have been applied to the case of inelastic particles in a constant gravitational field $[10,11,12]$. These systems often behave non intuitively. Hence, it is shown in [12] that a collection of inelastic particles confined to a vertical box with permanent injection of energy from the base (e.g. by shaking the base of the box vertically) the granular temperature profile is a non monotonic function of height. The temperature decreases near the bottom, goes through a minimum, and finally rises indefinitely with height above the minimum.

It has been recently shown in [13] that during the homogeneous regime of the temporal evolution of a one dimensional system of identical particles the spatial ordering of the particles appears to play a crucial role in 
shaping the particles' velocity distribution. Thus, works [14], based on the so-called pseudo-Maxwell model, a homogeneous model where no spatial ordering is assumed by construction, have been found to be unable to describe the evolution of a system of inelastically interacting particles, even during the homogeneous regime. The late evolution of systems of identically spatially ordered particles has been shown to be strongly affected by the development of spatial correlations between the particles' velocities eventually leading to an inelastic collapse [e.g. 2, 13].

One dimensional systems of identical particles behave in a very peculiar way. Thus, in the elastic limit, the overall velocity distribution of the particles' is not modified by collisions. Colliding particles do merely exchange their velocities and the system is not ergodic. Adjunction of just one anomalous particle forces the velocity distribution to relax towards a Maxwell-Boltzmann distribution.

In this paper we use molecular dynamics (MD) simulations to investigate the evolution of a two species onedimensional, periodic, system of $N$ point particles. $N / 2$ particles have mass $m_{1}=1$ (species 1 ) and $N / 2$ particles have mass $m_{2}=4$ (species 2 ). The system is a prototype for more complex systems with broader mass distributions. One important ingredient of the model is that colliding particles swap their relative position with a $50 \%$ probability allowing for the initial spatial ordering of the particles to evolve in time. This introduces the possibility of species to stream with respect to eachother giving frictional forces the opportunity to operate.

The restitution coefficient $r$, appearing in the collision rules (12) and (13), is close to elastic such that the thermalization time is always much shorter than the cooling time. As a consequence, the velocity distribution function of both species are always close to Maxwellian, at least as long as the species do not segregate. Such dissipative systems are always linearly unstable with respect to the so-called clustering instability. In one dimension the clustering instability is a fluid instability which is found to triggers the formation of non propagating spatial density inhomogeneities at scales of the order $10 \pi L / N \varepsilon$, where $\varepsilon \equiv 1-r^{2}$ and $L$ is the size of the system. The clusters of particles which form due to the clustering instability are characterized by a temperature profile which increases exponentially as a function of the particle index $l$ away from the center of the cluster (cf e.g. Figure 6). These strong temperature gradients within clusters drive the light particles away from their centers towards the edges of the clusters while the opposite happens to the heavy particles which tend to become concentrated in a very small region near the center of the cluster. (cf Figures 4 and 5). The species segregation is driven by the frictional force (7) which points in opposite directions for the light and heavy particles. At the time of inelastic collapse, fluid motions may or may not be supersonic depending on whether or not the number of particles $N$ exceeds a critical number of order $10 \pi / \varepsilon$. In order to avoid confusion, we do loosely define the time of inelastic collapse in our simulations as the time for which the molecular dynamic simulation is essentially "frozen". By "frozen" we mean that the total energy and, consequently the fluid velocity profiles of the system do not change significantly by doubling the number of collisions.

The paper is organized as follows. In Section 2 we introduce the set of fluid equations whereon we base our analysis of the simulation results. The numerical model is presented in Section 3. Simulations with $N=19600$ particles and 3 different values of the restitution coefficient $r$ are presented in Section 4, In Appendix A we develop the linear theory of the clustering instability for a twospecies, one dimensional system. The collision frequency for such a system in the homogeneous limit and close to the thermodynamic equilibrium, is given in Appendix B.

\section{Fluid equations for a mixture of two species}

In the general case [see e.g. 15], the expressions for the hydrodynamic transport coefficients describing a granular gas with two species of inelastically interacting particles are so involved that they are of little practical use to describe real systems. It is not even clear under which conditions fluid equations are relevant for real granular systems. However, over the years it has been shown that fluid equations are extremely useful to help understand results from granular system simulations. Over the few last decades, explicit expressions for the transport coefficients in 2 and 3 spatial dimensions have been published by various authors. To our knowledge, expressions for the $1 \mathrm{~d}$ case have not been published until now, certainly because of the non-ergodicity of most $1 \mathrm{~d}$ systems. Yet, in the case of a mixture of unequal particles, collisions among particles of different mass do inevitably drive the velocity distribution functions for all species towards Maxwell-Boltzmann. The tendency to thermalization in such one dimensional mixtures indicates that "standard" fluid equations may apply to such systems as well.

The fluid equations of this section all stem from a Chapman-Enskog type treatment of the Boltzmann equation by various authors $[7,8,15,9]$ which implies that their validity is at best limited by the assumptions on which the Chapman-Enskog procedure is based on. One of the most stringent conditions, in a system characterized by a mean free path $l_{\mathrm{mfp}}$ and a spatially varying temperature $T(x)$, is the requirement of the Knudsen number $K \equiv l_{\text {mfp }} \partial \log T / \partial x$ being much smaller than unity. The $K \ll 1$ condition means that the particles' distribution functions do not vary substantially over distances of the order of the mean free path $l_{\mathrm{mfp}}$ and that the velocity distributions are close to Maxwellian. A related condition is that fluid time scales must be long compared to the collisional time scale, i.e. the time between successive collisions of a thermal particle.

We write the continuity equation for two species $\alpha=$ 1,2 in terms of the species mean velocities $u_{\alpha}$ and mass densities $\varrho_{\alpha}=m_{\alpha} n_{\alpha}$ :

$$
\frac{\partial \varrho_{\alpha}}{\partial t}+\frac{\partial}{\partial x}\left(\varrho_{\alpha} u_{\alpha}\right)=0
$$


Defining the total density $\varrho \equiv \varrho_{1}+\varrho_{2}$ and the center of mass flow velocity $u \equiv\left(u_{1} \varrho_{1}+u_{2} \varrho_{2}\right) / \varrho$ one can add the two continuity equations (1) and write a one fluid continuity equation:

$$
\frac{\partial \varrho}{\partial t}+\frac{\partial}{\partial x}(\varrho u)=0 .
$$

According to [16] we write the momentum equation for both species in the form

$$
\varrho_{\alpha}\left(\frac{\partial u_{\alpha}}{\partial t}+u_{\alpha} \frac{u_{\alpha}}{\partial x}\right)=-\frac{\partial\left[p_{\alpha}\left(1+K_{\alpha}\right)\right]}{\partial x}+\phi_{\alpha}
$$

where $\phi_{\alpha}$ represents the frictional force on the species $\alpha$ due to the collisions with the other species in the system and where $K_{\alpha}$ is the contribution to the pressure tensor from collisions with all species (shear and bulk viscosity). Momentum conservation implies $\phi_{1}=-\phi_{2}$. The sign difference between $\phi_{1}$ and $\phi_{2}$ indicates that if the frictional force is dominant, it can act as a species filter. The partial pressures $p_{\alpha}$ in equation (3) are defined with respect to the center of mass flow velocity $u$, i.e.

$$
p_{\alpha} \equiv \int m_{\alpha}(v-u)^{2} f_{\alpha}(v) d v=n_{\alpha} T_{\alpha} .
$$

For the remaining of this section, and throughout the paper, we assume that collisions are efficient enough to ensure energy equipartition among species, i.e. $T=T_{1}=T_{2}$. This restriction implies the characteristic cooling time for each species being long with respect to the thermalization time. The energy equation for the mixture in the onedimensional case is then given by [15]

$$
\frac{\partial p}{\partial t}+u \frac{\partial p}{\partial x}+3 p \frac{\partial u}{\partial x}+2 \frac{\partial q}{\partial x}-\frac{8}{3} \eta\left(\frac{\partial u}{\partial x}\right)^{2}=-\zeta p
$$

where $p \equiv p_{1}+p_{2}$ is the total pressure, $q$ the total heat flux, $\eta$ the shear viscosity and $\zeta$ the cooling rate due to inelastic collisions. The bulk viscosity is zero by construction [see 9]. Similarly, the momentum equation for the mixture can be written in the conventional form [15]

$$
\frac{\partial u}{\partial t}+u \frac{\partial u}{\partial x}=-\frac{1}{\varrho} \frac{\partial}{\partial x}\left(p-\frac{4}{3} \eta \frac{\partial u}{\partial x}\right) .
$$

We note that this equation is equivalent to equations (3) up to terms which are quadratic in the species relative drift velocity $\delta u_{1} \equiv\left(u_{1}-u\right)$.

In the quasi-elastic limit, for small relative drift velocities, and assuming energy equipartition among species the following relations do conveniently close the above set of fluid equations. According to [16] the frictional force may be cast into the form

$$
\phi_{1}=a\left(n_{1}, n_{2}\right) 2 T \frac{n_{1} n_{2}}{n} \frac{\partial}{\partial x}\left[\ln \left(\frac{n_{1}}{n_{2}} T^{m_{12}}\right)\right]
$$

with $m_{12} \equiv\left(m_{2}-m_{1}\right) / m, m \equiv m_{1}+m_{2}$ and $n \equiv n_{1}+n_{2}$. In equation (7) $a$ is a dimensionless function of $n_{1}$ and $n_{2}$ which takes into account the species volume fraction and the radial distribution function of the contacting pairs. Explicit forms have been given for the 2 and 3 dimensional case [16]. In this paper we do not care about the exact form of equation 7 . We are just interested in the qualitative dependence of $\phi_{1}$ on the temperature $T$ and on the relative species concentration $n_{1} / n_{2}$. Following $[9,15]$ we write the constitutive relation for the heat flux as

$$
q=-\frac{n T}{\nu_{0} \sqrt{m_{1} m_{2}}} \frac{\partial T}{\partial x}\left[b_{1} \frac{n_{1}}{n} \sqrt{\frac{m}{m_{1}}}+b_{2} \frac{n_{2}}{n} \sqrt{\frac{m}{m_{2}}}\right]
$$

where we have defined the frequency $\nu_{0} \equiv V_{12} n \sigma$ and the characteristic velocity $V_{12}^{2}=2 \mathrm{Tm} /\left(m_{1} m_{2}\right)$, which, for species with Maxwellian distributions, is just the thermal velocity for the relative velocities between the two species (cf Appendix B). Again, both, $b_{1}$ and $b_{2}$, are rather involved functions of $n_{1} / n, m_{1}, m_{2}$ and even $V_{12}$, which we do not need to care about in this paper. In the above equation $\sigma$ has the dimension of a surface and may be interpreted as a collisional cross section. Formally, we express $\sigma$ in terms of the total number of particles $N$, the box length $L$ and the mean number density $\bar{n}$ :

$$
\sigma=\frac{N}{\bar{n} L} .
$$

With this definition, in a uniform system one has $\nu_{0}=$ $V_{12} N / L$. Starting from equation (27) of Jenkins and Mancini [7] we find that $\zeta$ must depend on the species densities via an expression of the kind

$$
\zeta=\varepsilon \nu_{0}\left[c_{11} \frac{n_{1}^{2}}{n^{2}} \sqrt{\frac{m_{2}}{m}}+c_{22} \frac{n_{2}^{2}}{n^{2}} \sqrt{\frac{m_{1}}{m}}+c_{12} \frac{n_{1} n_{2}}{n^{2}}\right]
$$

where the numerical coefficients $c_{11}, c_{12}$, and $c_{22}$ are unknown for the $1 \mathrm{~d}$ case and where $\varepsilon$ is the fractional energy loss per collision (see Section 3). Equation (10) is correct up to terms of order two in the gradients of hydrodynamic quantities $[8,15]$. Following Garzo et al $[15]$ we write the shear viscosity coefficient $\eta$ as

$$
\eta=\frac{n T}{\nu_{0}}\left[d_{1} \frac{n_{1}}{n} \sqrt{\frac{m}{m_{2}}}+d_{2} \frac{n_{2}}{n} \sqrt{\frac{m}{m_{1}}}\right]
$$

where, once more, $d_{1}$, and $d_{2}$ are order unity coefficients for which we do not care about the exact form. As a final remark, we note that in the set of fluid equations (2),(5) and (6) the inelasticity coefficient $\varepsilon$ does only appear in the energy equation (5) through the cooling rate $\zeta \propto \varepsilon \nu_{0}$. In particular, neither the friction $\phi_{1}$, nor the heat flux $q$ depend on $\varepsilon$, which is only acceptable in the quasi-elastic limit $\varepsilon \ll 1[15]$.

\section{Simulation model}

We use a one-dimensional model which is close to the traditional point-like hard particles on a ring model [13] with the notable difference that $N / 2$ particles have mass $m_{1}$ and $N / 2$ particles have mass $m_{2}$. In the strict one dimensional case the post collision velocities $v_{i}^{\prime}$ and $v_{j}^{\prime}$ for two 
Table 1. Basic parameters for the 3 simulations discussed in the paper. The total number of particles in each simulation is $N=19600$ with half of the particles with mass $m_{1}=1$ and the other half with mass $m_{2}=4$.

\begin{tabular}{l|l|l|l}
\hline Run & $\varepsilon$ & $r^{2}$ & $N \varepsilon$ \\
\hline 1 & $5 \times 10^{-4}$ & 0.9995 & 9.8 \\
2 & $2 \times 10^{-3}$ & 0.998 & 39.2 \\
3 & $2 \times 10^{-2}$ & 0.98 & 392 \\
\hline
\end{tabular}

colliding particles $i$ and $j=i+1$ of mass $m_{i}$ and $m_{j}$ and center of mass velocity $v_{0 i j}=\left(m_{i} v_{i}+m_{j} v_{j}\right) /\left(m_{i}+m_{j}\right)$ are given by

$$
\begin{aligned}
v_{i}^{\prime} & = \pm \frac{m_{j} v_{i j}}{m_{i}+m_{j}} r+v_{0 i j} \\
v_{j}^{\prime} & =\mp \frac{m_{i} v_{i j}}{m_{i}+m_{j}} r+v_{0 i j}
\end{aligned}
$$

where $r$ is the restitution coefficient $(r=1$ for elastic collisions), $v_{k}$ the velocity of particle $k=i, j$ before the collision and $v_{i j} \equiv v_{j}-v_{i}<0$ (we implicitly suppose a one dimensional axis $x$ with coordinates increasing from left to right $\left.x_{i}<x_{j}\right)$. With this definition of the restitution coefficient $r$ the kinetic energy of two colliding particles is reduced by a factor $\varepsilon \equiv 1-r^{2}$ in the center of mass frame. The + sign in (12) and the - sign in (13) correspond to the normal case where particle $i$ and $j$ do not exchange their relative positions during collision. The sign in (12) and the + sign in (13) correspond to the case where particles $i$ and $j$ swap their relative positions during collision. In the latter case, taking $r=1$, one has $v_{i}^{\prime}=v_{i}$ and $v_{j}^{\prime}=v_{j}$, i.e. particles just ignore each other. In principle one should reject this possibility as it is a non physical event for the case of a system of beads on a ring. We shall take advantage of this possibility as it allows for the different particles to become distributed spatially independently of the initial ordering with the possibility of species segregation to operate as in 2 and 3 dimensional systems.

\section{Simulation results}

In all simulations $N$ particles are initially distributed uniformly over the spatial domain $[0, L[$. We consider a simple two species system of $N / 2$ particles with mass $m_{1}=1$ and $N / 2$ particles with mass $m_{2}=4$. Particles of both species are initially disposed in alternating order. The initial velocity of a particle with mass $m_{\alpha}$ is randomly selected in the interval $[-0.5,0.5] / m_{\alpha}^{1 / 2}$ following a uniform probability distribution as in the [13] where the reference case of $N$ identical particles is discussed. The mean energy per particle is therefore the same for all species. Bulk velocities of all species are initially set to zero. The parameters for the 3 runs which will be discussed in the paper are listed in Table 1 . The somewhat arbitrary mass ratio $m_{2} / m_{1}=4$, which will be used throughout the paper, is sufficiently small to ensure efficient interspecies energy thermalization, so that we can assume $T_{1}=T_{2}$, and sufficiently large for the frictional force $\phi_{1}$ (see equation (7)) to play a non negligible role.

\subsection{Homogeneous phase}

The evolution of a one-dimensional system with not all particles having the same mass is very different from that of a one dimensional system of identical particles [e.g 6, $2,13]$. The difference is substantial, even in the elastic limit $r=1$. Indeed, while in the case of elastically interacting identical particles the velocity distribution does not change in time, the asymptotic velocity distribution is a Maxwell-Boltzmann velocity distribution when different species coexist in the system. The global thermalization rate strongly depends on the particles' mass distribution, each species $\alpha$ thermalizing at different rates depending on the species number density $N_{\alpha}$ and on the mass $m_{\alpha}$ of the species particles. For example in the case of a system with $N \gg 1$ and just one anomalous particle, the evolution towards a Maxwell-Boltzmann distribution is much slower than in the case of a two species system with $N / 2$ particles for each species, since only collisions among unequal particles can modify the velocity distribution functions in one dimensional systems.

In Figure 1 a snapshot of the velocity distribution function of both species are shown for Run 1 after a total number of $8 \times 10^{7}$ collisions . The distribution for both, light and heavy particles, which should be compared to the double peaked distribution of Figure 1 in [13] for the one species case, do closely follow a Maxwell-Boltzmann distribution disregarding of the fact that the initial distribution was strongly non thermal. This tendency of the velocity distributions to evolve towards Maxwell-Boltzmann

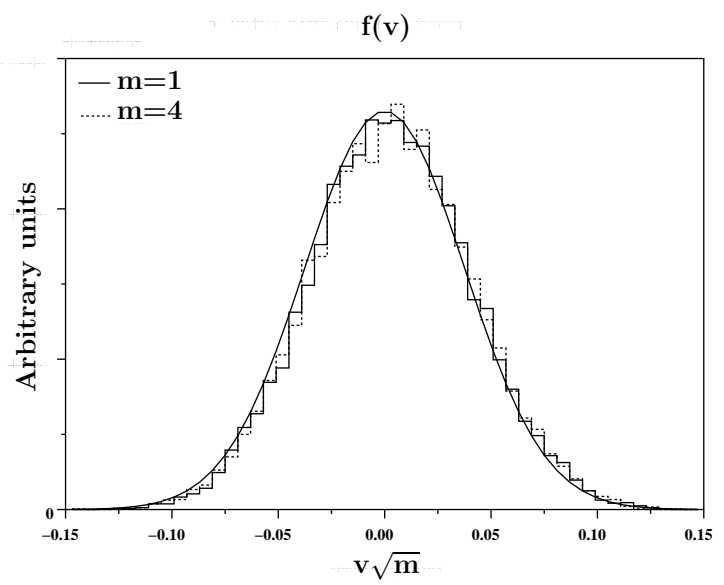

Fig. 1. Homogeneous regime for the case of $r^{2}=0.9995$ and $N=19600$ particles half of which have mass $m_{1}=1$ and the other half $m_{2}=4$ (Run 1 ). Shown are the velocity distribution histograms for both species after $8 \times 10^{7}$ collisions. The solid line represents the Maxwell-Boltzmann distribution corresponding to the measured particles' mean energy. 


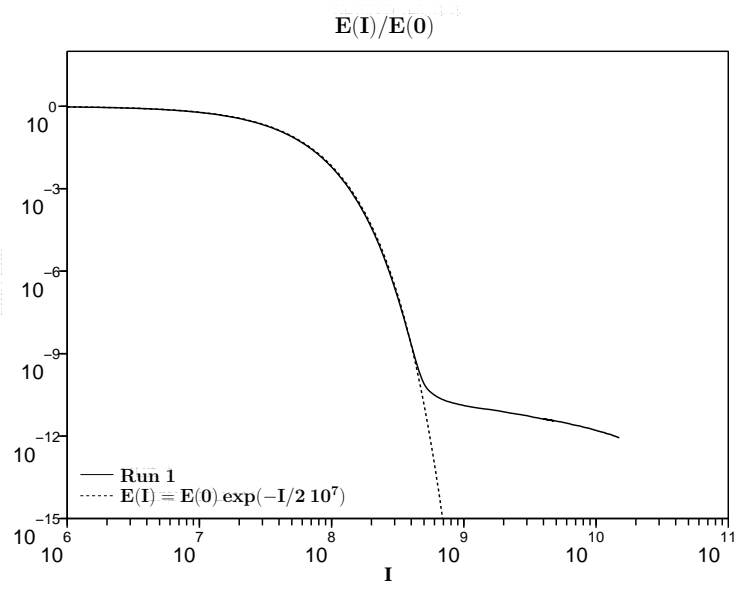

Fig. 2. Energy versus collision index $I$ for Run 1 . The exponential part of the profile closely follows the $E=E_{0} \exp (-I / 2 \times$ $10^{7}$ ) profile of the fluid theory.

allows the use of standard fluid theories $[7,8,15,9]$ to analyze the system's behavior.

Figure 2 shows the evolution of the energy in Run 1. The energy decreases exponentially as a function of the number of collisions $I$ during the first $510^{8}$ collisions. We shall define this phase as the homogeneous phase as it is perfectly well described by the homogeneous version of the fluid energy equation (5) (see equation (A.7) together with the relation (B.7)) which predicts a per collision energy loss of

$$
\frac{\overline{\delta E}}{E}=-2 \frac{\varepsilon}{N}
$$

Specifically, for $r^{2}=0.9995$ and $N=19600$ one has $\overline{\delta E} / E \approx 5.1 \times 10^{-8}$ which corresponds to the value $2 \times 10^{7}$ observed in Figure 2.

\subsection{Inhomogeneous phase}

After the initial homogeneous phase the system enter a new, inhomogeneous, regime where cluster formation becomes the dominant effect. The transition between the homogeneous and the inhomogeneous regime is clearly visible as a departure from the exponential energy decay in Figure 2. In this section we discuss the cluster structures observed in the simulations in the light of the fluid equations exposed in Section 2.

\subsubsection{Species segregation}

As in the one species case, the inhomogeneous regime in the multi species case is characterized by the presence of clusters of particles. Given the smallness of the inelasticity coefficient $\varepsilon$ and the initial homogeneity of the system, one expects the pressure to be spatially uniform for both species, except near the center of the collapsing cluster where the cooling rate $\zeta$ goes to infinity. Indeed, the temperature and density profiles, plotted for the light particles

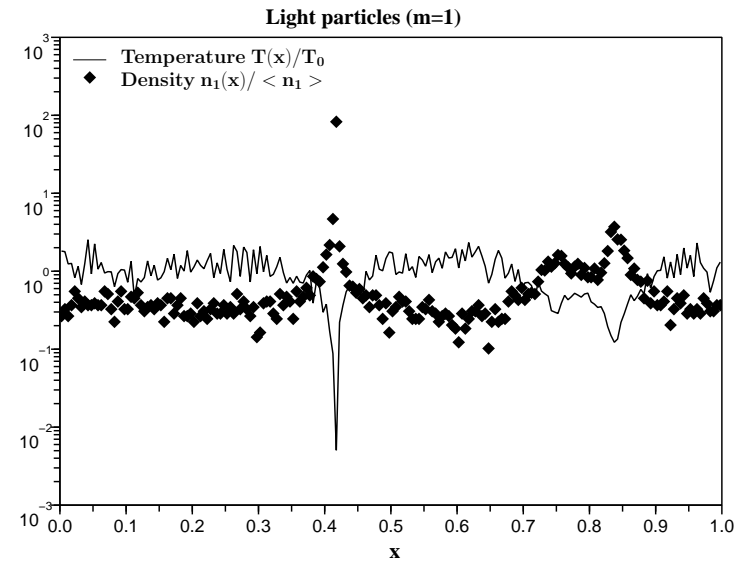

Fig. 3. Run 1: Temperature and density profiles for the light particles (i.e. $m=1$ ) normalized to their average value at the end of the simulation. Note the high concentration and low temperature within the cluster near $x=0.416$. Also note the presence of a second cluster forming near $x=0.8$.

at the end of Run 1 are seen to vary in antiphase (cf Figure 3) ensuring an approximate pressure balance.

However, light and heavy particles are not evenly distributed within the cluster. The relative concentration of light particles as a function of the particle index $l$ is shown in Figure 4 which clearly shows that the heavy particles are concentrated in the central part of the cluster, leaving an excess of light particles in the wings of the 6000 particles cluster.

In order to appreciate the spatial scales associated with the concentrations of light and heavy particles in the cluster we compute the integrated heavy particles excess func-

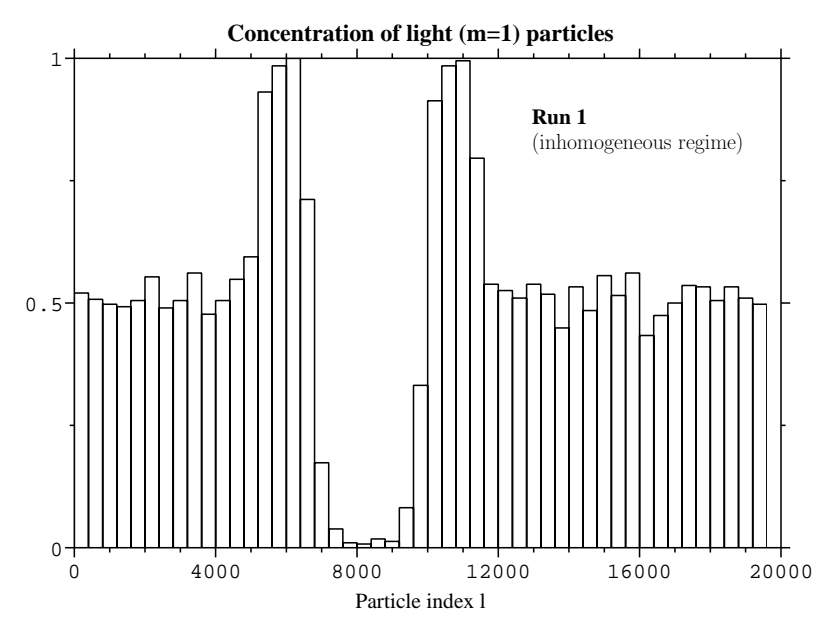

Fig. 4. Light particles histogram $(m=1)$ at the end of Run 1. Bin values represent the fractional number of light particles $n_{1} / n$. Each bin contains 400 particles. The main cluster includes all particles with index $l$ in the range 5000 to 11000 . 


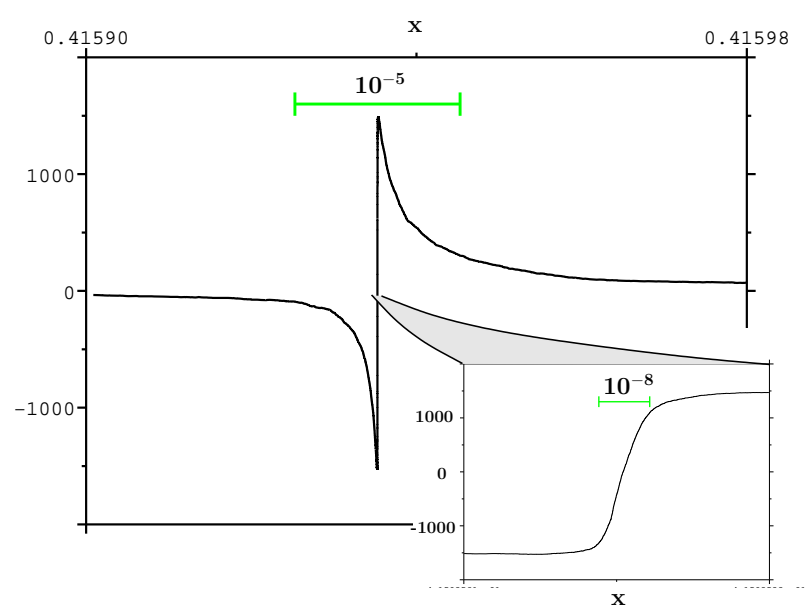

Fig. 5. Function $Y(x)$ (see equation (15)) for Run 1. The slope of the function $Y$ is a measure of the heavy (positive slope) or light (negative slope) particle excess.

tion $Y$ defined as

$$
Y(x)=\sum_{l=1}^{N}\left(\delta_{m_{l} m_{2}}-\delta_{m_{l} m_{1}}\right) \int_{0}^{x} \delta\left(\xi-x_{l}\right) d \xi
$$

Contribution to the integral is -1 for light particles $\left(m_{l}=\right.$ $1)$ and +1 for heavy particle $\left(m_{l}=4\right)$.

The function $Y(x)$ is plotted in Figure 5 and illustrates how disparate the spatial repartition of light and heavy particles are in the system. The core of the cluster contains some $3 \times 10^{3}$ heavy particles concentrated in a region of size $10^{-8}$. From Figure 4 we know that the $3 \times 10^{3}$ light particles of the cluster are largely excluded from this small central region dominated by the heavy particles but Figure 5 shows that the light particles dominate over a much larger region of size $10^{-5}$, the characteristic size of the cluster at the end of the simulation. The species segregation is the visible effect of the frictional force $\phi_{1}$ (cf equation (7)) of the two fluid momentum equations (6). In case of equal densities $n_{1}=n_{2}$ and a spatially varying temperature profile $T(x)$ the frictional force $\phi_{1} \propto\left(m_{2}-m_{1}\right) \partial T / \partial x$ points up the temperature gradient for the light species $m_{1}$ and down the temperature gradient for the heavy species $m_{2}$. Segregation appears as a ineluctable consequence of the temperature gradients within clusters.

\subsubsection{Temperature profiles in clusters}

Figure 3 shows that the temperature in a cluster strongly decreases towards its center. Figure 6 shows the temperature profiles in the inhomogeneous regime for Run 1 and 2. The figure illustrates the fact that the temperature drops exponentially as a function of the particles index $l$ towards the center of the cluster. In these particular cases the temperature decreases by several order of magnitudes from edge to center. As expected, the thermalization time being

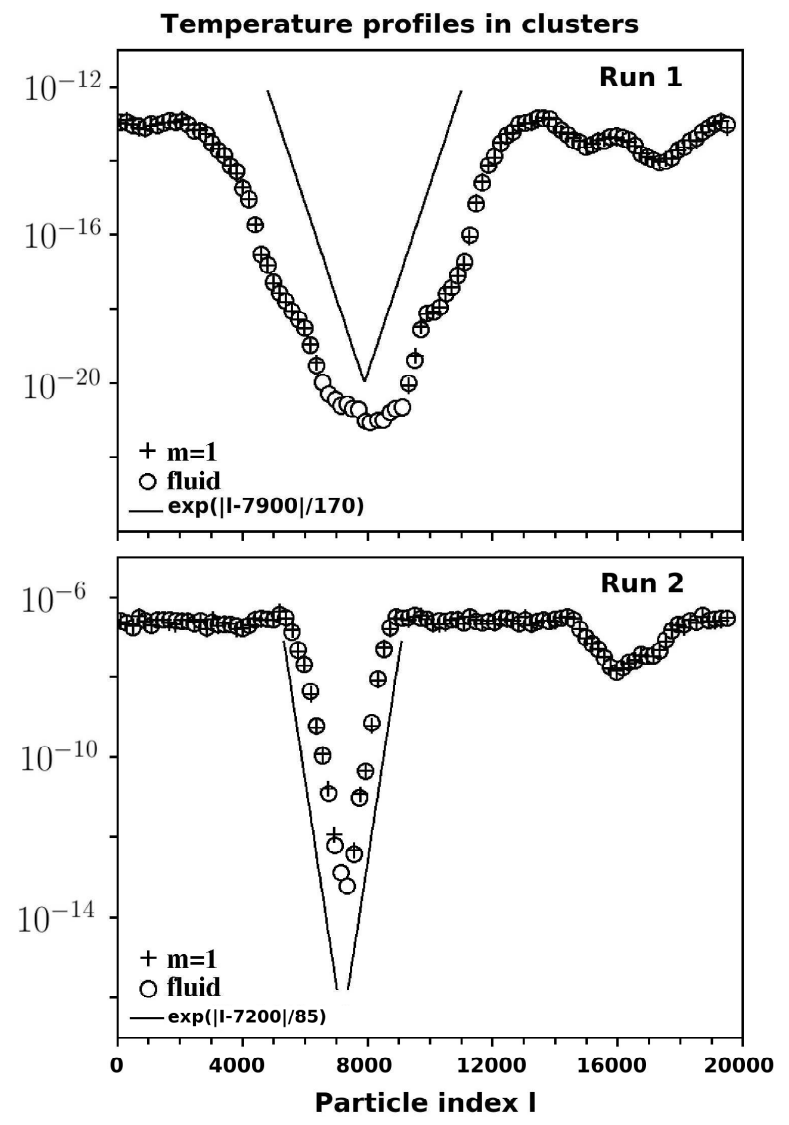

Fig. 6. Fluid temperature (circles) and light particles' temperature (plus) as function of the particle index for Run 1 (top panel) and Run 2 (bottom panel) during the inhomogeneous regime. In both simulations the temperature is found to decrease exponentially towards the center of the cluster according to a characteristic scale of order $\varepsilon^{-1 / 2}$.

much shorter than the cooling time, the fluid and light particle temperature profiles closely follow each other. The interesting point is that the granular temperature decreases exponentially towards the center of the cluster with a scale which seems to be of order $\varepsilon^{-1 / 2}$. Again, the $\varepsilon^{-1 / 2}$ scaling of the temperature profile in Fig. 6 can be deduced easily from the fluid equations. In order to do so, we evaluate the temperature profiles within clusters in the static limit. Using the energy equation (5) we can write

$$
2 \frac{\partial q}{\partial x}=-\zeta p
$$

Let us suppose that in a given region of the system species 1 is the dominating one, i.e. $n \approx n_{1} \gg n_{2}$. In this case the heat flux reads (cf equation (8))

$$
q=-\frac{b_{1}}{\sigma}\left(\frac{T}{2 m_{1}}\right)^{1 / 2} \frac{\partial T}{\partial x}
$$

whereas the right hand side of Eq. (16) becomes

$$
-\zeta p=-\varepsilon c_{11} \sigma\left(\frac{2 T}{m_{1}}\right)^{1 / 2} T n^{2}
$$


and the static energy equation (16) writes

$$
\frac{\partial}{\partial x}\left(T^{1 / 2} \frac{\partial T}{\partial x}\right)=\varepsilon \frac{c_{11}}{b_{1}} \sigma^{2} T^{-1 / 2} p_{0}^{2}
$$

where we have used the static limit $p=p_{0}=$ const of the momentum equation (6). In order to compute the temperature profile as a function of the particle index $l$ we have to introduce the transformation from the spatial coordinate $x$ to the particle index $l$.

$$
l(x)=A \int_{0}^{x} n(\xi) d \xi
$$

where $A$ is a surface such that $A n(\xi) d \xi$ is the number of particles in the interval $[\xi, \xi+d \xi]$. Using the transformation (20) we write the energy equation (19) in terms of the particle index $l$

$$
\frac{\partial}{\partial l}\left(T^{-1 / 2} \frac{\partial T}{\partial l}\right)=\frac{\varepsilon}{4} \frac{c_{11}}{b_{1}} T^{1 / 2}
$$

where we have used the fact that the particles' cross section $\sigma$ is equal to $A / 2$ given the $50 \%$ swap probability for colliding particles. The solution of $(21)$ is

$$
T \propto \exp \left\{ \pm l\left(\varepsilon \frac{c_{11}}{2 b_{1}}\right)^{1 / 2}\right\}
$$

If $c_{11}$ and $b_{1}$ are numerical constants of order unity, it follows, as already suggested by Figure 6 , that the typical scale of variation for the temperature profile is of order $\varepsilon^{-1 / 2}$

\subsection{Cluster formation and linear fluid theory}

As in one species simulations [6], Figure 7 shows that at the time of inelastic collapse, the number of clusters is an increasing function of the $N \varepsilon$. Even the Mach number of the fluid motions is seen to depend on $N \varepsilon$, the simulation with the smallest value of $N \varepsilon$ being characterized by subsonic motions and the two other simulations being characterized by supersonic motions with the evident formation of shocks. As pointed out by Ben-Naim et al [17] the piecewise linear velocity profiles observed in Figure 7 are a direct consequence of the momentum equation (6). Indeed, as already discussed in the context of Figure 3 , the pressure in the system is essentially constant everywhere except within clusters (shocks in the supersonic regime). In this case the momentum equation reduces to $\varrho(\partial u / \partial t+u \partial u / \partial x)=0.75 \eta \partial^{2} u / \partial x^{2}$ with solutions of the type $u(x, t)=u_{0}+\left(x-x_{0}\right) / t$, where $u_{0}$ and $x_{0}$ are integration constants.

The difference between the velocity profiles at the time of inelastic collapse for the three runs shown in Figure 7 can be explained on the basis of a linear analysis of the fluid equations of Section 2 developed in Appendix A. The linearized system of equations has three eigenmodes,

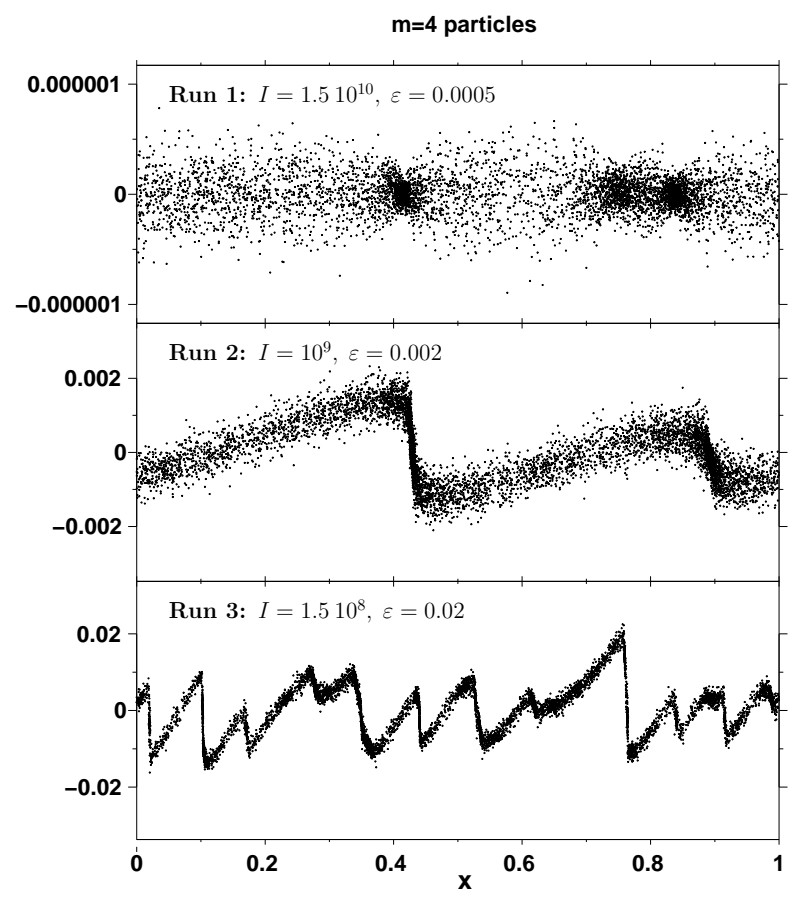

Fig. 7. Inhomogeneous regime: scatter plot for the $m=4$ particles for all runs at the time of inelastic collapse.

two of which correspond to the right and left propagating sound wave and the non propagating (zero real frequency) entropy mode. The main difference with respect to classical gas dynamics is that all modes are unstable at long wave length. The other important prediction of linear theory is that in general, but more specifically for wave vectors $k$ such that $\tilde{k} \equiv k L /(N \varepsilon) \lesssim 1$, the non propagating entropy mode grows substantially faster than the sound mode. Indeed, the growth rate of the entropy mode always peaks at $k=0$ whereas the sound mode's growth rate vanishes as $k \rightarrow 0$. A careful analysis of the growth rates does actually shows that the entropy mode is always the fastest growing mode (see Appendix A).

\subsubsection{The dominant mode}

What determines the number of clusters in a one dimensional system at the time of inelastic collapse? Knowledge of the initial conditions for the velocity fluctuations of all modes makes it possible to estimate different characteristic time scales in terms of the dimensionless (pseudo) time variable $\tau$ (defined in A.5) using the approximations of linear theory.

Initially, in all systems, particles are distributed uniformly in the spatial interval [0,1[ with $N / 2$ particles of mass $m_{1}=1$ and $N / 2$ particles of mass $m_{2}=4$. The velocity of a particle of mass $m$ is sorted randomly in the velocity interval $[-0.5,0.5] / m^{1 / 2}$ so that the average kinetic 
energy per particle is the same for all species corresponding (formally) to an initial temperature $T_{0}=1 / 12$ and thermal velocity $v_{0}^{2}=2 /(6 \bar{m})=1 / 15$. Thermalization of such a system takes only a few collisions per particle so that all species can be assumed to be initially distributed according to a Maxwellian distribution at temperature $T_{0}=1 / 12$, $\varepsilon$ being much smaller than unity. The initial amplitudes of the velocity fluctuations can be evaluated using the Wiener-Khintchine theorem which states that the power spectrum of the fluid velocity field for a set particles with uncorrelated velocities is flat (white noise). The immediate consequence of the Wiener-Khintchine theorem is that energy is evenly distributed among all Fourier modes, i.e. all modes have equal amplitude $\left|\delta u_{0}\right|$. Requiring the total kinetic energy of all particles in the system being $N T / 2$ one must have

$$
\delta u_{0}^{2}=\frac{T}{\bar{m}} \frac{4}{N}=2 \frac{v_{0}^{2}}{N}
$$

In all simulations, at $t=0$, we have $T=1 / 12, N=19600$, $m_{1}=1$ and $m_{2}=4$. The initial amplitude of the velocity fluctuations are therefore of order $\delta u_{0}=2.6 \times 10^{-3}$ with $v_{0}=0.258$, which corresponds to $\delta U_{0}=(2 / N)^{1 / 2}=1.01 \times$ $10^{-2}$.

From linear theory we know that at small values of the wave vector $\tilde{k} \lesssim 1 / 2$ the growth rate is approximately given by $\gamma N / \varepsilon=1-\tilde{k}^{2} / 2$ indicating that the mode with the longest possible wavelength is always the fastest growing mode. It is then expected that clustering always occurs at the largest possible scale allowed by the system with only one cluster growing in the system. This is effectively the case in Run 1 only but not in Run 2 (with 2 clusters) and particularly in Run 3 where several clusters occur. This can be understood by noting that linear theory predicts that the density fluctuation of the entropy mode is proportional to the wave vector $k$ :

$$
\frac{\delta n_{k}}{n}=-i \tilde{k} \delta U_{k}
$$

where $\delta U_{k} \equiv \delta u_{k} / v_{0}$ is essentially the fluctuation's Mach number. Thus, for two modes $k_{1}$ and $k_{2}$ with equal initial velocity fluctuation amplitude $\delta U_{k 1}=\delta U_{k 2}$, the one with the largest wave vector is the one with the largest associated density fluctuation and therefore a more likely candidate for triggering the inelastic collapse, provided the growth rate of the two modes are not too dissimilar. For example, in Run 3, there are some 10 visible density clusters corresponding to an average distance between clusters of the order $\lambda=L / 10$. Therefore, the mode which triggers the inelastic collapse has $\tilde{k}=0.16$ and a linear growth rate which is only roughly $1 \%$ less than the growth rate of the smallest possible wave vector $\tilde{k}_{\min } \equiv 2 \pi / N \varepsilon$, but, according to equation 24 , with a 10 times larger density fluctuation. Thus, as long as the velocity fluctuation amplitude of the mode $\tilde{k}=0.16$ is not much smaller than the velocity fluctuation amplitude of the fastest growing mode, i.e. potentially during the first $100 \gamma^{-1} \approx 100 \mathrm{~N} / \varepsilon=9.8 \times 10^{7}$ collisions, the strongest density fluctuations in the system are due to the mode with $\tilde{k}=0.16$. Indeed, in Run
Table 2. Characteristic time scales of the entropy mode for the smallest possible wave vector $k=k_{\min } \equiv 2 \pi / N \varepsilon$. The column labelled Supersonic indicates whether or not the fluid motions are supersonic at the time of inelastic collapse. $\gamma$ refers is the linear growth rate of the mode, $\tau_{\mathrm{s}}$ and $\tau_{\mathrm{n}}$ are defined in equations (25) and (26), respectively. The parameters of the runs are given in Table 1.

\begin{tabular}{c|c|l|l|l|l}
\hline Run & Supersonic & $\tilde{k}_{\min }$ & $\gamma^{-1}$ & $\tau_{\mathrm{s}}$ & $\tau_{\mathrm{n}}$ \\
\hline 1 & no & 0.64 & $4.9 \times 10^{7}$ & $2.3 \times 10^{8}$ & $2.5 \times 10^{8}$ \\
2 & yes & 0.160 & $1.0 \times 10^{7}$ & $4.6 \times 10^{7}$ & $6.4 \times 10^{7}$ \\
3 & yes & 0.016 & $1.0 \times 10^{6}$ & $4.5 \times 10^{6}$ & $8.6 \times 10^{6}$ \\
\hline
\end{tabular}

3 particle clustering and supersonic motions are already present after $I \approx 4 \times 10^{6}$ collisions, which, using the definition for $\tau$ given by (B.7) in Appendix B corresponds to $\tau=2.06 I \approx 8 \times 10^{6}$. This time is too short by more than one order of magnitude for the fastest growing mode to become dominant in terms of density fluctuations.

We conclude that, for quasi elastic restitution coefficients $\varepsilon \ll 1$, the mode which dominates the inelastic collapse has a wave vector $\tilde{k} \sim 1 / 5$ corresponding to a wavelength $\lambda \sim 10 \pi L / N \varepsilon$. If such a long wavelength does not fit into the system, the dominant mode is simply the one with the longest possible wavelength, i.e. $\lambda=L$.

\subsubsection{Supersonic fluid motions}

Let us now address the question of the magnitude of the characteristic fluid velocities at the time of the inelastic collapse. Velocities are subsonic in Run 1 and supersonic in Runs 2 and 3.

We introduce the sonic time scale $\tau_{\mathrm{s}}$, defined as the time for the amplitude of the velocity fluctuation $\delta U_{k}$ to reach unity assuming linear growth, i.e.

$$
\tau_{\mathbf{s}}(\tilde{k})=-\frac{\ln \left(\delta U_{0}\right)}{\gamma(\tilde{k})} .
$$

For a system of uniformly distributed particles with uncorrelated velocities, the initial fluctuation amplitude is $\delta U_{0} \approx(2 / N)^{1 / 2}$. Similarly, using equation (24) we define $\tau_{\mathrm{n}}$ as the time for the density fluctuation $\delta n(k) / n$ to reach an amplitude of order unity, i.e.

$$
\tau_{\mathrm{n}}=-\frac{\ln \left(\tilde{k} \delta U_{0}\right)}{\gamma(\tilde{k})}
$$

Both, $\tau_{\mathrm{s}}$ and $\tau_{\mathrm{n}}$ are listed in Table 2 for the mode with the longest wavelength $\tilde{k}_{\text {min }}$.

In Run 1 the motions remain subsonic at the time of the inelastic collapse despite the simulation lasting significantly longer than $\tau_{s}$, meaning that supersonic motions had enough time to develop. We conclude that an inelastic collapse is not necessarily associated with supersonic motions. The explanation at hand can be found in Table 2 which shows that in Run 1 the characteristic times $\tau_{\mathrm{s}}$ 
and $\tau_{\mathrm{n}}$ are very much the same for the dominant mode $k=k_{\min } \equiv 2 \pi / N \varepsilon$ while the sonic time scale $\tau_{\mathrm{s}}$ is significantly shorter than $\tau_{\mathrm{n}}$ for the dominant mode in Runs 2 and 3. From Table 2 it follows that supersonic motions develop before the occurrence of an inelastic collapse provided $\tilde{k}_{\text {min }} \lesssim 1 / 5$, i.e. $10 \pi \lesssim N \varepsilon$.

\section{Conclusions}

We have performed simulations of a one dimensional two species periodic system of $N$ point particles undergoing inelastic collisions. In order to keep the possibility for the system to arrange particles independently of the initial configuration, particles are allowed to change their relative positions during collisions with a $50 \%$ probability. Initial velocities for particles of species $\alpha$ are selected randomly according to a constant distribution in the range $[-0.5,0.5] / m_{\alpha}^{1 / 2}$. Unlike one dimensional systems of identical particles, where the homogeneous regime is characterized by double peaked velocity distributions [13, 18], one dimensional multi-species systems tend to evolve towards a Maxwell-Boltzmann distribution. This tendency justifies the use of "standard" fluid equations to describe such systems. As for one dimensional systems of identical particles, the inhomogeneous regime is characterized by the formation of clusters which eventually make an inelastic collapse. It has been shown in [2] that clustering in one species systems is driven by a beam instability. We show that in a two species system clustering is triggered by a fluid instability of the non propagating entropy mode, for which density and temperature fluctuations vary in antiphase ensuring spatial pressure balance. The sound mode is also found to be unstable but its growth rate is always substantially smaller than the growth rate of the entropy mode. Because of the non propagating nature of the entropy mode, the one dimensional system does evolve naturally towards a series of clusters with symmetric temperature and density profiles and antisymmetric velocity profiles.

The number of particles in the clusters is determined by the number of particles in one wavelength of the dominant mode of the clustering instability, i.e. the mode which first reaches a relative density fluctuation of order unity. The typical wavelength of this mode has been found to be of the order $\lambda \approx 10 \pi L / N \varepsilon$ corresponding to $10 \pi / \varepsilon$ particles per cluster. Clustering is generally characterized by supersonic fluid motions unless the total number of particles $N \lesssim 10 \pi / \varepsilon$.

A peculiar aspect of the late evolution of a multispecies systems is the appearance of species segregation within the collapsing clusters, heavy particles becoming concentrated in an extremely small region around the center of the cluster and the light particles filling the space between the central region and the edges of the cluster. Segregation is driven by the temperature gradient inside the cluster [19] via the frictional force $\phi$ in the one-species fluid momentum equation (3).
We thank both referees for the many pertinent and helpful comments which strongly helped us to improve the original manuscript.

\section{A Linear theory for a one-dimensional system}

In this appendix we develop the linear theory for the clustering instability in a one-dimensional and spatially uniform system of inelastically colliding particles using the set of fluid equations of Section 2. Assuming particles of mean mass $\bar{m}$, we write the set of equations pertinent to the one-dimensional one-fluid case as

$$
\begin{aligned}
\frac{\partial n}{\partial t}+u \frac{\partial n}{\partial x}+n \frac{\partial u}{\partial x} & =0 \\
\bar{m}\left(n \frac{\partial u}{\partial t}+n u \frac{\partial u}{\partial x}\right)+\frac{\partial}{\partial x}\left(n T-\frac{4}{3} \eta \frac{\partial u}{\partial x}\right) & =0 \\
\frac{\partial}{\partial t}(n T)+u \frac{\partial}{\partial x}(n T)+3 n T \frac{\partial u}{\partial x} & \\
+2 \frac{\partial q}{\partial x}-\frac{8}{3} \eta\left(\frac{\partial u}{\partial x}\right)^{2}+c \varepsilon \frac{N}{L} v_{0} T n & =0
\end{aligned}
$$

where $c$ is a constant of order unity, which will be computed explicitly for a two species system in Appendix B. The other quantities in equation (A.3) are defined as

$$
\begin{aligned}
v_{0} & \equiv\left(\frac{2 T}{\bar{m}}\right)^{1 / 2} \\
\eta & \equiv d \frac{n T}{\nu_{0}}=d \frac{n T}{v_{0}} \frac{L}{N} \\
q & \equiv-b \frac{n T}{\nu_{0} m} \frac{\partial T}{\partial x}=-\frac{b}{2} n v_{0} \frac{L}{N} \frac{\partial T}{\partial x}
\end{aligned}
$$

with $b$ and $d$ being two more constants of order unity. We note that for a two species system the normalization velocity $v_{0}$ is related to the thermal velocity $V_{12}$ of the species relative velocity of Section 2 via

$$
V_{12}^{2}=2 \frac{\bar{m}}{m_{1} m_{2}} v_{0}^{2} .
$$

The linear analysis of the system is easier if performed in terms of the dimensionless variables $\tau$ and $U$ :

$$
\begin{aligned}
d \tau & =N^{2} \frac{v_{0}}{L} d t \\
U & =\frac{u}{v_{0}} .
\end{aligned}
$$

where $d \tau / d t$ is a measure of the number of collisions per time unit in the system during the homogeneous phase and $U$ is essentially the flow's Mach number.

We assume a static equilibrium $u(x, \tau)=0$ with a uniform density $n=n_{0}$, and temperature $T(x, \tau)=T_{0}(\tau)$ decreasing according to equation (A.3), i.e.

$$
\frac{\partial T_{0}}{\partial \tau}=-c \frac{\varepsilon}{N} T_{0} \rightarrow T_{0}(\tau) \propto e^{-c \varepsilon \tau / N} .
$$


During the linear phase of the instability $c$ is a constant which only depends on the species relative densities and on the species particle mass (see Appendix B). For example, if $N / 2$ particles have mass $m_{1}=1$ and $N / 2$ particles $m_{2}=4$, one has $c=0.97$. Thus, $c=0.97$ must be used for all runs discussed in the paper except Run 1, where all particles are identical and for which the linear theory presented here is not applicable.

Let us now investigate the response of the equilibrium characterized by $n=n_{0}$ and $T=T_{0}(\tau)$ to small perturbations $\delta n, \delta \theta$ and $\delta U$ by setting

$$
\begin{aligned}
n & =n_{0}+\delta n \\
T & =T_{0}(1+\delta \theta) \\
U & =\delta U .
\end{aligned}
$$

The coefficients of the resulting linear system are independent of $\tau$ and $x$ so that we can solve it using standard Fourier techniques. We therefore assume perturbations of the type $\delta n, \delta \theta, \delta U \propto \exp [i(k x-\omega \tau)]$ which, when plugged into the linearized system, lead to

$$
\mathbf{M} \cdot\left(\begin{array}{c}
\delta n / n_{0} \\
\delta U \\
\delta \theta
\end{array}\right)=0 .
$$

where $\mathbf{M}$ is the $3 \times 3$ matrix given by

$$
\mathbf{M} \equiv\left(\begin{array}{ccc}
-i \tilde{\omega} & i \tilde{k} & 0 \\
i \tilde{k} & \frac{4}{3} D \tilde{k}^{2}-2 i \tilde{\omega}-2 & i \tilde{k} \\
2-i \tilde{\omega} & 3 i \tilde{k} & B \tilde{k}^{2}-i \tilde{\omega}
\end{array}\right)
$$

with $\tilde{k} \equiv k L /(N \varepsilon), \tilde{\omega} \equiv \omega N / \varepsilon, B \equiv \varepsilon b$ and $D \equiv \varepsilon d$. Non trivial solutions of the system correspond to a vanishing determinant of the matrix M. Splitting the complex frequency $\omega=\omega_{r}+i \gamma$ into real and imaginary parts $\omega_{r}$ and $\gamma$, and assuming real wave vectors $k$ leads to two equations for the real and the imaginary part of the condition $\operatorname{det} \mathbf{M}=0$ (in the remaining of this appendix we drop the tildes to ease readability):

$$
\begin{aligned}
& 2\left(1-B k^{2}-\frac{2}{3} D k^{2}-3 \gamma\right) \omega_{r}^{2} \\
& +B k^{4}+3 \gamma k^{2}-2 \gamma^{2}+2 \gamma^{3}-2 k^{2}-2 B \gamma k^{2} \\
& +2 B \gamma^{2} k^{2}+\frac{4}{3} D \gamma^{2} k^{2}+\frac{4}{3} D B \gamma k^{4}=0
\end{aligned}
$$

and either

$$
\begin{aligned}
\omega_{r}^{2}= & 3\left(\gamma^{2}+\frac{k^{2}}{2}\right)-2 \gamma\left(1-B k^{2}-\frac{2}{3} D k^{2}\right) \\
& -B k^{2}+\frac{2}{3} B D k^{4} .
\end{aligned}
$$

for the modified sound wave, or

$$
\omega_{r}=0
$$

for the modified, non propagating, entropy wave. The dispersion relation for sound waves, $\omega_{r}^{2}=3 k^{2} / 2$, is immediately obtained by setting $\gamma=B=D=0$ in equation (A.14) (we shall remember that frequencies are measured in terms of the pseudo time $\tau$ and not the real time t). Switching back to real time using equation (A.5), the dispersion relation reduces to the more familiar relation $\omega_{r}^{2}=3 T k^{2} / \bar{m}$ for a one dimensional system. The growth rate $\gamma(k)$ for both, sound and entropy mode, can be obtained by substituting $\omega_{r}^{2}$ from equation (A.14) or (A.15), respectively, into (A.13). After some juggling one ends up with

$$
\begin{aligned}
f_{s}(\gamma, & k, B, D)=16 \gamma^{3}-16\left(1-\frac{2}{3} D k^{2}-B k^{2}\right) \gamma^{2} \\
& -\left(12 B k^{2}-8 B D k^{4}-4 B^{2} k^{4}-\frac{16}{9} D^{2} k^{4}\right. \\
& \left.+\frac{16}{3} D k^{2}-6 k^{2}-4\right) \gamma \\
& -2 B^{2} k^{4}+\frac{4}{3} B^{2} D k^{6}+\frac{8}{9} B D^{2} k^{6}-\frac{8}{3} B D k^{4} \\
& +2 B k^{4}+2 B k^{2}+2 D k^{4}-k^{2}=0
\end{aligned}
$$

for the sound mode, and

$$
\begin{aligned}
& f_{e}(\gamma, k, B, D)=2 \gamma^{3}+\left(2 B k^{2}+\frac{4}{3} D k^{2}-2\right) \gamma^{2} \\
& \quad+\left(3 k^{2}-2 B k^{2}+\frac{4}{3} B D k^{4}\right) \gamma \\
& \quad+B k^{4}-2 k^{2}=0
\end{aligned}
$$

for the entropy mode. For small values of $B$ and $D$, i.e. at sufficiently large spatial scales such that both thermal conduction time scale $1 / B k^{2}$ and the viscous time scale $1 / D k^{2}$ both exceed the linear time scale $1 / \omega$, one may use the asymptotic expressions for the imaginary part of both the sound wave and the entropy wave, viz.

$$
\begin{aligned}
f_{s}(\gamma, k, 0,0) & =16 \gamma^{3}-16 \gamma^{2} \\
& +\left(6 k^{2}+4\right) \gamma-k^{2}=0 .
\end{aligned}
$$

and

$$
\begin{aligned}
f_{e}(\gamma, k, 0,0) & =2 \gamma^{3}-2 \gamma^{2} \\
& +3 k^{2} \gamma-2 k^{2}=0 .
\end{aligned}
$$

We note that $f_{s, e}(0, k, 0,0)<0$ for all values of $k$. Since $f_{s, e}(\gamma \rightarrow \infty)=\infty$, a positive real solutions $\gamma>0$ must exist for both equations (A.18) and (A.19). Thus, both the sound and the entropy mode are always unstable for $k \rightarrow 0$. There is indeed, only one real solution of (A.18) which goes as $k^{2}$ for $|k| \rightarrow 0$ and tends towards the asymptotic value $1 / 6$ for $|k| \rightarrow \infty$. Similarly, for $|k| \rightarrow \infty$, the entropy mode's growth rate tends towards $2 / 3$. Of course, for sufficiently large values of $k$ both modes must be damped by diffusive effects. However, unlike the entropy mode, which is always unstable for $|k| \rightarrow 0$, the sound mode is completely stabilized for thermal diffusivity values $B>1 / 2$. 


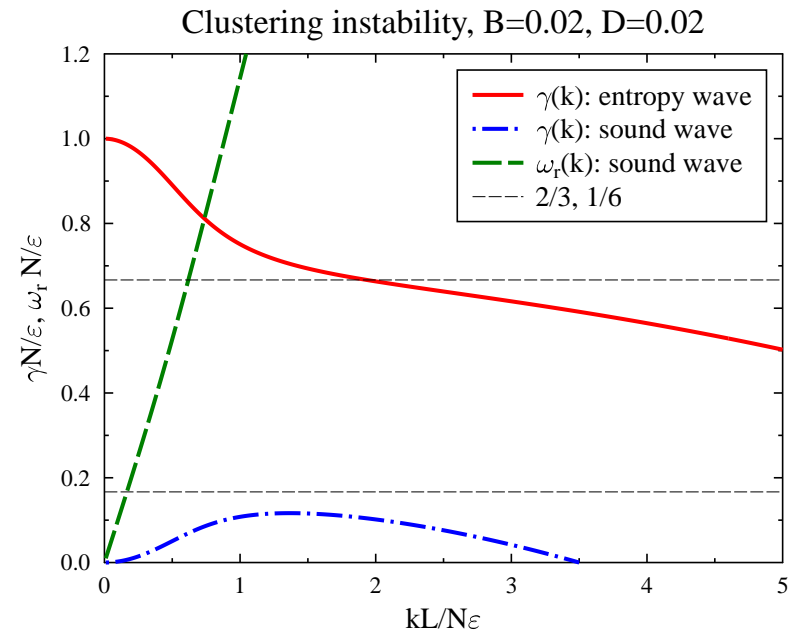

Fig. 8. Growth rate for the both the sound mode and the entropy mode for a particular value of the thermal diffusion coefficient $B$ and the shear viscosity coefficient $D$. Plotted is also the real frequency of the sound mode $\omega(k)$ and the asymptotic values of the growth rates for both modes in the limit $k \gtrsim 1$, and $D=B=0$.

The fact that the entropy mode is a non propagating mode implies that density and temperature fluctuations vary spatially in antiphase, ensuring pressure balance. Indeed, for $k \rightarrow 0$, one has $-i \omega=1-k^{2} / 2$ (see Figure 8) from where, using the second line of the matrix in equation (A.11), one immediately deduces the linear pressure equilibrium condition $\delta n / n_{0}=-\delta \theta$ which is valid up to order one in $k$. To same order in $k$, the first equation in (A.11) implies the $\delta n / n_{0}=-i k \delta U$. This latter relation shows that in the entropy mode density and velocity fluctuations are out of phase by $\pi / 2$ corresponding to the fluid flowing from the low density to the high density parts of the wave. These linear motions, eventually enforced by non linear effects (i.e. velocity profile steepening) at late times, ineluctably drive the system toward the final inelastic collapse.

\section{B Collision frequency for Maxwellian distributions}

The number of collisions per time unit of a particle of species $\alpha$ with the particles of species $\beta$ for relative velocities in the range $[u, u+d u]$ is given by

$$
d \nu_{\alpha \beta}=|u| f_{\alpha \beta}(u) d u, \quad u<0 .
$$

In equation (B.1) $f_{\alpha \beta}(u)$ denotes the distribution of the relative velocities. If one assumes Maxwellian distributions for both species, the distribution of the relative velocities is also Maxwellian. In one dimensions, with $N_{\beta}$ particles uniformly distributed in a system of length $L$ it must be that

$$
f_{\alpha \beta}(u)=\frac{1}{V_{\alpha \beta} \pi^{1 / 2}} \frac{N_{\beta}}{L} e^{-u^{2} / V_{\alpha \beta}^{2}}
$$

where $V_{\alpha \beta}$ is the thermal velocity defined as

$$
V_{\alpha \beta}^{2} \equiv V_{\alpha}^{2}+V_{\beta}^{2}=2 \frac{T_{\alpha}}{m_{\alpha}}+2 \frac{T_{\beta}}{m_{\beta}} .
$$

Integration of equation (B.1), assuming the Maxwellian distribution (B.2), gives the collision frequency of a particle $\alpha$ with the particles of species $\beta$ :

$$
\nu_{\alpha \beta}=\int_{-\infty}^{0}|u| f_{\alpha \beta}(u) d u=\frac{N_{\beta}}{L} \frac{V_{\alpha \beta}}{2 \pi^{1 / 2}} .
$$

The total collision frequency $\nu$ in the system is obtained by adding the contributions from all kinds of collisions, i.e.

$$
\begin{aligned}
\nu= & N_{\alpha} \nu_{\alpha \beta}+N_{\beta} \nu_{\beta \alpha}+N_{\alpha} \nu_{\alpha \alpha}+N_{\beta} \nu_{\beta \beta}= \\
& 2 \frac{N_{\alpha} N_{\beta}}{L} \frac{V_{\alpha \beta}}{2 \pi^{1 / 2}}+\frac{N_{\alpha}^{2}}{L} \frac{V_{\alpha \alpha}}{2 \pi^{1 / 2}}+\frac{N_{\beta}^{2}}{L} \frac{V_{\beta \beta}}{2 \pi^{1 / 2}}
\end{aligned}
$$

Assuming energy equipartition $T=T_{\alpha}=T_{\beta}$ and an equal number of particles for both species $N_{\alpha}=N_{\beta}=N / 2$, one ends up with

$$
\begin{aligned}
\nu\left(m_{\alpha}, m_{\beta}, T, N\right)= & \frac{N^{2}}{4 L} \frac{v_{0}}{\pi^{1 / 2}}\left[\left(\frac{\bar{m}}{m_{\alpha}}+\frac{\bar{m}}{m_{\beta}}\right)^{1 / 2}+\right. \\
& \left.\left(\frac{\bar{m}}{2 m_{\alpha}}\right)^{1 / 2}+\left(\frac{\bar{m}}{2 m_{\beta}}\right)^{1 / 2}\right]
\end{aligned}
$$

where $\bar{m} \equiv\left(m_{\alpha}+m_{\beta}\right) / 2$ and $v_{0}^{2} \equiv 2 T / \bar{m}$. Using the expression for the collision frequency given by (B.6) it becomes possible to specify the constant $c$ which establishes a relation between the time variable $t$ and the collision index $I$ (cf equations (A.3) and (A.7)). Of course, given the above assumptions, the relation between $t$ and $I$ only holds as long as the inhomogeneities in the system are weak. By comparing (A.7) and (14) one obtains the following relation between the pseudo time variable $\tau$ and the collision index $I$ :

$$
2 \delta I=c \delta \tau
$$

Given the relation between $\tau$ and the time variable $t$ (see equation (A.5)) and using the fact that the collision frequency is just $\nu=\delta I / \delta t$ it follows that

$$
\begin{aligned}
c \equiv 2 \nu \frac{L}{N^{2} v_{0}}= & \frac{1}{2 \pi^{1 / 2}}\left[\left(\frac{\bar{m}}{m_{\alpha}}+\frac{\bar{m}}{m_{\beta}}\right)^{1 / 2}\right. \\
& \left.+\left(\frac{\bar{m}}{2 m_{\alpha}}\right)^{1 / 2}+\left(\frac{\bar{m}}{2 m_{\beta}}\right)^{1 / 2}\right]
\end{aligned}
$$

We note that $c$ does not depend on the temperature, as long as energy equipartition is a valid approximation. 


\section{References}

1. H. M. Jaeger, S. R. Nagel, and R. P. Behringer. Granular solids, liquids, and gases. Reviews of Modern Physics, 68:1259-1273, October 1996.

2. S. McNamara and W. R. Young. Kinetics of a onedimensional granular medium in the quasielastic limit. Phys. Fl., 5:34-45, January 1993.

3. I. Goldhirsch and G. Zanetti. Clustering instability in dissipative gases. Physical Review Letters, 70:16191622, March 1993.

4. E. Efrati, E. Livne, and B. Meerson. Hydrodynamic Singularities and Clustering in a Freely Cooling Inelastic Gas. Physical Review Letters, 94(8):088001-+, March 2005.

5. B. Bernu and R. Mazighi. One-dimensional bounce of inelastically colliding marbles on a wall . Journal of Physics A Mathematical General, 23:5745-5754, December 1990.

6. S. McNamara and W. R. Young. Inelastic collapse and clumping in a one-dimensional granular medium. Phys. Fluids, 4:496-504, March 1992.

7. J. T. Jenkins and F. Mancini. Kinetic theory for binary mixtures of smooth, nearly elastic spheres. Physics of Fluids, 1:2050-2057, December 1989.

8. J. J. Brey, J. W. Dufty, C. S. Kim, and A. Santos. Hydrodynamics for granular flow at low density. Phys. Rev. E, 58:4638-4653, October 1998.

9. V. Garzó and J. W. Dufty. Hydrodynamics for a granular binary mixture at low density. Phys. Fluids, 14:1476-1490, April 2002.

10. J. Eggers. Sand as Maxwell's Demon. Physical Review Letters, 83:5322-5325, December 1999.

11. J. Javier Brey, F. Moreno, R. García-Rojo, and M. J. Ruiz-Montero. Hydrodynamic Maxwell demon in granular systems. Phys. Rev. E, 65(1):011305-+, January 2002.

12. R. Ramírez and R. Soto. Temperature inversion in granular fluids under gravity. Physica A Statistical Mechanics and its Applications, 322:73-80, May 2003.

13. A. Baldassarri, U. Marini Bettolo Marconi, and A. Puglisi. Influence of correlations on the velocity statistics of scalar granular gases. Europhysics Letters, 58:14-20, April 2002.

14. E. Ben-Naim and P. L. Krapivsky. Multiscaling in inelastic collisions. Phys. Rev. E, 61:5-+, January 2000.

15. V. Garzó, J. M. Montanero, and J. W. Dufty. Mass and heat fluxes for a binary granular mixture at low density. Physics of Fluids, 18:3305-+, August 2006.

16. J. T. Jenkins and D. K. Yoon. Segregation in Binary Mixtures under Gravity. Physical Review Letters, 88(19):194301-+, May 2002.

17. E. Ben-Naim, S. Y. Chen, G. D. Doolen, and S. Redner. Shocklike Dynamics of Inelastic Gases. Physical Review Letters, 83:4069-4072, November 1999.

18. D. Benedetto, E. Caglioti, and M. Pulvirenti. A Kinetic equation for granular media. Math. Mod. Num. Anal., 31(5):615-641, 1997.
19. S. S. Hsiau and M. L. Hunt. Granular thermal diffusion in flows of binary-sized mixtures. Acta Mech., 114(1):121-137, March 1996. 